\title{
小学数学教学有效阅读审题的研究
}

谢先琼

湖北省荆州市洪湖市峰口镇明德小学

DOI:10.32629/jief.v2i6.1014

[摘 要] 数学是小学一门重要的基础课程, 提高学生的数学学习能力和做题能力对学生的成绩与以后的发展来说都大有益处。对于小学的学 生来说, 所学的数学知识和内容难度加大, 尤其对学生的审题能力有较高要求。有的学生明明掌握了相应的数学定理, 但总解不对题目, 很 大一方面原因就是审题能力较差, 没有明确题目考查的重点是什么。因此, 在小学数学教学中, 教师要着重培养和提高学生的数学审题能力, 做好解题的先导才能顺利解答, 获得良好的数学成绩。

[关键词] 小学学生; 数学阅读审题能力; 培养探究

中图分类号: G633.6 文献标识码: A

数学可以启迪我们的智慧、开阔我们的思维, 数学可以使我们的生 活更加绚丽多彩。我们不仅应当重视数学教学, 还应当重点培养学生利 用数学知识解决应用题的能力。

\section{1 数学审题能力的重要性}

数学题目由多种语言组成, 数学审题需对各种语言及其关系进行认 真思考。从而制订正确的解题计划。阅读审题时应学会用数学语言表达, 用数学方法解决问题。数学审题能力是一种综合能力, 其主要体现在能 认真分析提供的数学阅读材料, 能从材料中寻找有用的数学信息。能对 数学阅读材料进行整合, 正确找出解答问题的方法。能利用所学知识解 决生活中的问题。发展实际解决问题的能力。

解题是小学阶段检测学生数学学习水平的重要手段。解答数学题中, 需学生动脑动手解决问题。通过解题有利于学生掌握基础的数学知识, 提升学生的数学思维。合理有效地审题, 使学生用正确的数学思维认真 读懂题意, 找到最佳的解题方法, 大大提高学习效率。

\section{2 小学数学阅读审题现状}

在小学数学的教学过程中, 让学生掌握阅读审题的技巧是小学数学 学习内容的重要一部分。小学教材中, 应用题占到了很大的一部分, 如 果不具备阅读审题的能力, 就不可能正确解答应用题。应用题是来源于 我们的生活, 但又比实际生活更难, 所以必须让学生熟练的掌握解题的 方法、技巧等, 笔者认为, 多数的小学生在审题方面都存在以下的问题:

2.1 多数学生审题态度很不端正, 觉得审题就是粗略的浏览一遍题 意, 对题目中的重点很难把握, 对题目中隐含的条件就是掌握不清, 对 题目中的题意理解不清, 导致自己对题目无从下手, 跟自己之前掌握的 数学知识联系不上。

2.2 学生在审题的过程中, 对于关键词的掌控不准。要想正确的解 答一道题, 就必须要抓住题目的重点, 问题是什么, 我们就去解决什么, 带着这样的疑问去仔细阅读题目, 找到自己觉得有用的关键信息, 就可 以辅助学生更好的去理解题意。但往往大多数学生在平时的数学学习的 过程中, 没有形成去仔细读题的好习惯, 多数学生存在读完了题目却仍 然对题目的要求很模糊, 严重阻碍了自己的数学学习。

2.3 学生在审题的时候, 不能够灵活的联系上之前学过的数学知识 点。学生在审题的过程中, 所面对的最大问题是题目中所要用到的知識, 无法与所学的数学知识进行完美的链接, 学生往往不知道该用什么样的 知识点, 用什么样的方法去解决问题。

2.4 学生的审题方式太过固化, 很不灵活。学生往往不能做到举一 反三, 教师在教课的过程中, 稍微改变一下题意学生就不知所措, 教师 通常是教会了学生解决一类型题目的方法, 学生在解题的过程中, 对于 运用那种方法解题理解不清, 导致解题困难。

\section{3 小学生有效阅读审题的措施}

3.1 教师应注重阅读审题方法的教授
古人说 “给人以鱼不如授人以渔” 只有教会学生解题的思路和方法, 才能使学生真正的掌握数学的知识, 使学生能够在做题时达到举一反三。 所以, 在小学数学教学的过程中, 教师必须注重对学生审题技巧的培养。

3.2 让学生注重读题

学生学习数学的时候, 必须注重对自己阅读审题能力的培养。学会 读题是一切的前提。在学生的传统思维里, 对于读题很不重视, 所以往 往在做数学题目的过程中, 往往是简单的看一下题目, 就刎忙着手去做 题, 没有系统性的思考。有些学生首先会去先读问题, 然后带着问题去 题目中找到与问题相关的关键信息, 这样从问题入手, 不仅可以节省时 间, 更可以提高自身解决数学问题的质量, 达到事半功倍的效果。在审 题的过程中, 不能一味的求快, 更重要的是应该从内心深处, 认识到读 题的作用, 从题目中快速的抓取到关键的信息。读题不一定要大声的朗 读出来, 而是要在内心里细细的品味。

3.3 抓住题目中的重点

学生在审题的过程中, 往往对题目中的重点不能够很好的把握, 找 不到题目中隐含的信息。学生经常会忽略题目中关键的信息和问题, 小 学的数学中, 出现最多的就是 “谁比谁多, 谁比谁少, 谁比谁快, 谁比 谁慢” 等问题, 就涉及到了倍数等问题, 教师会通常在这些题目中给学 生设置陷阱, 如果不能很好的对题目进行阅读, 抓住题目中关键的信息 就很可能掉入教师的陷阱当中去。因此, 教师在平时的课堂教学中, 必 须锻炼学生在审题时对题目中重点的把控。题目中, 内容太长, 隐含的 条件太多, 就需要学生在题目中做一些标记, 方便自己解题时重点的思 考、探索。

\section{4 总结}

总的来说, 教师在数学教学的过程中, 注重对学生阅读审题能力的培 养, 至关重要, 不仅可以增强学生学习数学的信心, 而且可以培养学生学 习数学的兴趣, 将之前被动的学习转化为主动的探索、求知, 数学的成绩 就自然而然会得到提升, 也为以后数学的学习打下了坚实的基础。目前, 小学数学阅读能力的缺失, 直接导致了学生数学成绩的提升, 所以注重数 学阅读审题的培养至关重要。就目前而言, 有效审题阅读当前仍面临的很 多问题, 培养学生有效阅读的能力, 需要教师们在平时的授课过程中逐渐 的去培养, 不断的根据学生的特点, 对自己的教学方法进行改进、提升, 在提高自己教学质量的同时, 也培养了学生学习数学的兴趣。

\section{[参考文献]}

[1]张泽旭.浅谈如何有效地进行小学数学的课堂导入 [J]. 新课 程,2020(38):15.

[2]马晓骋. 小学数学教学学具操作有效性的研究 [J]. 新课 程,2020(38):18.

[3] 蔺淑霞. 分析小学数学实践活动课教学探讨 [J]. 新课 程,2020(38):67. 